Narváez Burbano, J. H., y Obando Guerrero, L. M. (mayo-agosto, 2021). Relación entre factores predisponentes a la deprivación sociocultural y el apoyo social en adolescentes. Revista Virtual

Universidad Católica del Norte, (63), 39-62. https://www.doi.org/10.35575/rvucn.n63a3

\title{
Relación entre factores predisponentes a la deprivación sociocultural y el apoyo social en adolescentes
}

\begin{abstract}
Relation between predisposing factors to sociocultural deprivation and social support in adolescents
\end{abstract}

\section{Jonathan Harvey Narváez Burbano}

Doctor en Ciencias de la Educación

Programa de Psicología, Universidad de Nariño

Pasto, Colombia

jonnathanharnavaez@gmail.com

Orcid: https://orcid.org/0000-0002-3023-5156

CvLac: https://scienti.minciencias.gov.co/cvlac/visualizador/generarCurriculoCv.do?cod_rh=0001437688

\section{Lina María Obando Guerrero}

Magister en Psicodiagnóstico y Evaluación Psicológica

Programa de Psicología, Universidad de Nariño

Pasto, Colombia

linamariaobandoguerrero@gmail.com

Orcid: https://orcid.org/0000-0001-6286-5251

CvLac: https://scienti.minciencias.gov.co/cvlac/visualizador/generarCurriculoCv.do?cod rh=0000072358

Recibido: 15 de mayo de 2020

Evaluado: 8 de noviembre de 2020

Aprobado: 26 de febrero de 2021

Tipo de artículo: Investigación Científica y Tecnológica

\section{Resumen}

Diversos estudios dan cuenta de la relación existente entre los sistemas nucleares (familia, escuela, comunidad) y el desarrollo cognitivo, social y emocional de los individuos en los diversos contextos. El artículo tuvo como objetivo establecer la relación entre los factores predisponentes a la deprivación sociocultural y el apoyo social en adolescentes. El estudio fue cuantitativo, de tipo 
no experimental, descriptivo- correlacional y transversal; para la recolección de la información se aplicó la versión adaptada del Cuestionario de Apoyo Social-AS y la Escala de Factores Predisponentes a la Deprivación Sociocultural, a una muestra de 350 estudiantes, cuyas edades oscilaban entre los 13 y 18 años, provenientes de escuelas públicas en los ambientes rural y urbano. Para el análisis de datos se utilizó el software SPSS. Se encontró que en la mayoría de participantes el nivel de deprivación sociocultural y apoyo social es medio y que existía una relación inversa estadísticamente significativa entre las variables, lo que indica que a mayor apoyo social menor exposición a los factores predisponentes a la deprivación sociocultural, de manera que las fuentes de apoyo instrumental, emocional o informacional, en los ámbitos familia, escuela y comunidad, reduce la ocurrencia de manifestaciones de deprivación sociocultural, como comportamientos antisociales, deficiente adaptación a los entornos culturales y bajo nivel en el rendimiento académico.

Palabras clave: Adolescencia; Apoyo social; Deprivación sociocultural; Escuela - comunidad.

\begin{abstract}
Various studies show the relationship between nuclear systems (family, school, community) and the cognitive, social and emotional development of individuals in the various contexts. The article aimed to establish the relationship between factors predisposing to sociocultural deprivation and social support in adolescents in the city of Pasto, Colombia. The study was quantitative, nonexperimental, descriptive-correlational and transversal. To collect the information, the adapted version of the Social Support Questionnaire-AS and the Sociocultural Deprivation Scale were applied to a sample of 350 students aged between 13 to 18 years from public schools in rural and urban settings. The SPSS software was used for data analysis. It was found that in the majority of participants the level of socio-cultural deprivation and social support is medium and that there is a statistically significant inverse relationship between the variables, which indicates that a greater social support reduced exposure to the factors predisposing to socio-cultural deprivation, so that the sources of instrumental, emotional or informational support in the family environment, school, community reduces the occurrence of manifestations of socio-cultural deprivation such as
\end{abstract}


antisocial behaviors, poor adaptation to cultural environments and low level of academic performance.

Key words: Adolescence; Social support; Sociocultural deprivation; School - community.

\section{Introducción}

El individuo como sujeto social está rodeado de diversos condicionantes ambientales, los cuales, al estar asociados a factores socio-relacionales, influyen en el desarrollo cognitivo, social y emocional de niños, niñas y adolescentes (NNA). La situación de deprivación sociocultural (DS) es definida como una capacidad reducida que tienen los individuos "para modificar sus estructuras intelectuales y responder a las fuentes externas de estimulación” (Cedillo, 2010, p. 29). Su génesis está sujeta a un conjunto de circunstancias que obstaculizan el desarrollo cognitivo-social de los individuos, al estar asociadas a los diversos ambientes de socialización, donde NNA obtienen herramientas para el desarrollo y estimulación cognitiva. Es así como los contextos socialmente deprivados, son aquellos en cuya esfera social y relacional no logran proveer las herramientas necesarias para la estimulación cognitiva y socioemocional, que permita a los individuos un afrontamiento efectivo ante a los diversos factores de riesgo y demandas sociales.

Para Cabezas (2017) la deprivación sociocultural representa una condición con prevalencia en las personas que viven inmersas en ambientes de pobreza cultural y/o material, para quienes los procesos de transmisión cultural están subordinados por la reproducción de prácticas de sociabilidad violenta y precariedad económica, las cuales cumplen una función adaptativa y socializadora, en el marco de los procesos de interacción y deficientes redes de apoyo. Es así como logran identificarse algunos factores predisponentes a situaciones de deprivación sociocultural en las esferas familiares, comunitarias y escolares, las cuales actúan como predictores de las manifestaciones de DS (Narváez, 2019).

Al respecto, Jiménez (2009) considera a la DS como un conjunto de circunstancias que van a impedir o dificultar el normal desarrollo cognitivo, físico, emocional y/o social de las personas. Esta condición es considerada por Lundber (1998) como una deficiente interacción dinámica entre las disposiciones genéticas y el medio ambiente en el que se desarrolla el niño, especialmente en 
una sociedad incapaz de proporcionar importantes fuentes de estimulación al universo de su población. A esto Fuica et al. (2014) añaden que la DS es una carencia en la transmisión cultural por razones internas o por las condiciones ambientales en las que se ve inmerso el sujeto, lo que lleva a un estado de desventaja observable y demostrable que se relaciona con la familia, comunidad o sociedad a la cual pertenece el individuo o grupo, acarreando una serie de manifestaciones que dificultan los procesos académicos, las dinámicas adaptativas y el desarrollo de sus proyectos de vida (Barello et al., 2007); de allí la importancia de lograr sociedades más igualitarias, las cuales sean socialmente más cohesionadas y que estimulen la comunicación y el diálogo de saberes (Falla et al., 2016), es decir, que favorezcan los vínculos de confianza entre los miembros y la participación en la vida comunitaria (Mena et al., 2018).

En efecto, tal como lo proponen Thomas \& Shihadeh (2013), la privación estructural de recursos y una cultura cívica participativa débil fomentan el aislamiento institucional entre los jóvenes, lo que, a su vez, eleva las tasas de delincuencia. En ese sentido, el apoyo social comunitario, sumado a vínculos institucionales sólidos son esenciales en la transmisión cultural, lo que facilita la internalización de los valores, el fortalecimiento de lazos de redes locales y comportamientos pro-sociales, minimizando el riesgo a la deprivación sociocultural. Sin embargo, lo individuos que carecen de tales fuentes de apoyo, se exponen a factores de riesgo que desencadenan desventajas cognitivas, sociales y culturales respecto a la población general, y en casos más específicos una afectación a la salud, el desarrollo cognitivo y la adaptación social (Mena et al., 2018).

Entre las manifestaciones de deprivación sociocultural, García (2003) identifica algunas deficiencias en los procesos, como el registro de ideas, la utilización de estrategias, la secuenciación de ideas, la organización general del texto y la falta de revisión en sus textos (ortografía y caligráfica), sumada a procesos desadaptativos y baja motivación. Asimismo, Sánchez (2010), al estudiar a un grupo de niños provenientes de contextos deprivados socioculturalmente, señala que el nivel cognitivo de los niños era limítrofe, inferior al CI promedio, razón por la cual su ritmo de aprendizaje era lento, y presentaban disfuncionalidad cognitiva y comportamental. Entre los rasgos más significativos subraya dificultades específicas como problemas de expresión, escritura y cálculo, del mismo modo problemas en la adaptación a 
sistemas normativos, dificultad en la relación con adultos y conflicto entre pares, siendo evidentes los comportamientos disruptivos dentro del ambiente educativo.

Las manifestaciones mencionadas se desencadenan cuando algunos factores predisponentes a la deprivación sociocultural actúan sobre el entorno de desarrollo del NNA, obstaculizando la adquisición de herramientas o recursos, debido a las inadecuadas o inoperantes fuentes de apoyo instrumental, emocional o informacional (Narváez, 2019). Los factores predisponentes a la DS están asociados a los ámbitos familiar, comunitario y escolar (Cedillo, 2010). El ámbito familiar es el sistema más influyente en el desarrollo del NNA, al ser la familia el espacio donde NNA se desarrollan socialmente, demarcando el andamiaje de las funciones cognitivas y socializadoras que determinan el desenvolvimiento en otros escenarios de interacción (Suarez y Vélez, 2018); entre los factores predisponentes a la DS, en este ámbito, están el clima y la dinámica familiar.

El clima social familiar hace referencia al estado de bienestar entre los miembros de una familia, lo que incluye el grado de comunicación, el control e influencia entre ellos, en función de las dimensiones de relaciones, desarrollo y estabilidad (Estévez et al., 2008; Reyes et al., 2019). Mientras la dinámica familiar corresponde al juego de subjetividades, entorno a las normas, reglas, límites, jerarquías y roles, mediante las cuales se regula la convivencia y se establecen pautas que armonizan la vida familiar (Gallego et al., 2019).

En el ámbito comunitario, se identifica el apoyo social comunitario, el cual se concibe como la disponibilidad de ayuda entre las personas, grupos y organizaciones que conforman una unidad residencial o barrio, y atiende dimensiones como la integración, participación y apoyo de los sistemas formales en la comunidad (López y Herrera, 2019). Y en el ámbito escolar, el clima social escolar, referido al conjunto de características psicosociales de un centro educativo, permeadas por las dinámicas interpersonales, estructurales y funcionales, las cuáles pueden facilitar u entorpecer los procesos de adaptación, interacción y aprendizaje de los miembros de una comunidad educativa, donde se buscan nuevos amigos, se reafirma la identidad y se logra reconocimiento social (Gómez González et al, 2017). Entre las dimensiones del clima social escolar que pueden ser predisponentes a la deprivación sociocultural están las relaciones pedagógicas, el tipo de convivencia, la cohesión, las normas y creencias (Gálvez et al., 2018). 
Para Ruiz y Merino (2009) dentro de la realidad de los contextos educativos, se relaciona y atribuye el éxito o fracaso escolar a las limitaciones del entorno familiar, es decir, que la deprivación cultural en estos contextos está en función del análisis de las carencias que tienen los alumnos frente a la socialización primaria en el entorno familiar; sin embargo, Narváez (2019) sostiene que no sólo el ámbito familiar repercute en la forma como el individuo se aprovisiona de herramientas y recursos que permitan su socialización y desarrollo. Es allí donde el apoyo social se constituye en un constructo fundamental, al momento de comprender cómo los sistemas familiar, educativo y escolar proveen o no las condiciones informativas, afectivas o instrumentales, para el desarrollo del individuo en los contextos cotidianos (Orcasita y Uribe, 2010).

El concepto del apoyo social puede entenderse desde tres perspectivas: estructural, funcional y contextual; a nivel estructural comprende el conjunto de provisiones instrumentales, bien sean reales o percibidas, del individuo a la comunidad o personas significativas; en lo funcional hace referencia a las aportaciones materiales, informacionales y emocionales que un individuo recibe de su red social; mientras que la perspectiva contextual, centra su interés en las condiciones situacionales del apoyo social percibido, siendo este un predictor del bienestar de las personas (Uribe, 2016). Al respecto, existe un importante acervo teórico e investigativo que relaciona el apoyo social con los factores predisponentes a la deprivación sociocultural.

Aranda y Pando (2013) señalan que el apoyo social tiene como funcionalidad la reducción de las consecuencias de las demandas y estresores sociales del individuo, y asegura condiciones de facilitación que permitan la mejora de la calidad de vida. Hombrados-Mendieta \& Castro-Travé (2013) proponen la relación entre apoyo social, clima escolar y percepción de conflictos; Barcelata et al. (2013), por su parte, plantean una relación significativa entre el funcionamiento familiar, la dinámica familiar y el apoyo social, al momento de afrontar escenarios de riesgo psicosocial; mientras Zambrano y Almeida (2017), en su estudio entre clima familiar y conducta violenta, refieren que el apoyo social o la falta de apoyo es un determinante al momento de evaluar el grado de cohesión de la familia, y un predictor fundamental de los comportamientos agresivos en el hogar.

En efecto, el presente artículo contribuye al análisis de los factores predisponentes de la deprivación sociocultural, en relación con el apoyo social, cuyo estado de conocimiento a nivel latinoamericano, pese a la relación significativa entre las variables, no cuenta con evidencias 
investigativas que le den sustento. A su vez, se busca ofrecer insumos que permitan retroalimentar las políticas públicas en educación, desarrollo comunitario y exclusión social; fundamentando el desarrollo de los individuos, en función de factores relacionales y ambientales que facilitan o dificultan el acceso a herramientas y recursos que determinan la desigualdad y desventajas socioafectivas y de apoyo entre los individuos en un mismo contexto.

\section{Metodología}

El presente fue un estudio cuantitativo, de tipo no experimental, descriptivo- correlacional y transversal. Se plantea que fue no experimental dado que no hubo una manipulación de las variables y se observó el fenómeno tal como se da en su contexto natural (Hernández et al., 2010). Por su parte, fue descriptivo - correlacional porque midió, caracterizó y relacionó las variables de apoyo social y factores predisponentes a la deprivación sociocultural. Finalmente, fue transversal dado que se evaluaron las variables en un momento determinado; de este modo, los datos se recogieron en un tiempo único (Hernández et al., 2010).

\section{Muestra}

La muestra de tipo no probabilístico intencional estuvo constituida por 350 estudiantes de secundaria de una institución educativa pública, cuyas edades oscilaban entre los 13 y 18 años. Para la selección de la muestra se tomaron como criterios de inclusión que fuesen estudiantes entre los 13 y 18 años, que estuvieran con matrícula vigente en una institución educativa pública, que procedan de los centros urbanos, periféricos y rurales en los estratos socioeconómicos 1 , 2 y 3 , en los grados escolares de básica secundaria y media, y que tuvieran debidamente diligenciados el asentimiento y consentimiento informado. Por su parte, se excluyeron aquellos casos que no tenían este rango de edad, que no cursaban los grados 6 a 11, que pertenecieran a estratos socioeconómicos distintos al 1, 2, 3, y aquellos que no tuviesen firmado el consentimiento y asentimiento informado. 


\section{Técnicas e instrumentos de recolección de información}

Cuestionario de Apoyo Social- (Matud, 1998)

Se aplicó la versión adaptada del cuestionario de apoyo social -AS (Villalobos, 2009) que recoge información sobre la percepción global de apoyo social disponible, en función de la disponibilidad de personas que pueden prestar apoyo emocional, instrumental e informacional en las áreas personal, laboral y familiar. El cuestionario estuvo constituido por 13 ítems, de tipo Likert, cuyas respuestas se puntúan de la siguiente manera: 1-Nunca, 2- Rara vez, 3- Algunas veces, 4- Casi siempre y 5- Siempre. La versión adaptada en Colombia quedó constituida por un factor y la consistencia interna fue de 0.9.

Escala de factores predisponentes a la deprivación sociocultural (FAPDESO) (Narváez, 2019)

La FAPDESO fue elaborada en Colombia y evalúa el grado de predisposición de un individuo a los factores de deprivación sociocultural. Quedó constituida por 18 ítems que se encuentran distribuidos en cuatro factores, correspondientes al social comunitario, dinámica familiar, clima escolar y el clima social familiar. La escala es de tipo Likert y las opciones de respuesta: 1- totalmente en desacuerdo, 2- desacuerdo, 3- de acuerdo y 4- totalmente de acuerdo.

\section{Procedimiento}

Inicialmente, se solicitó la autorización de las instituciones educativas para el desarrollo de la investigación, para lo cual se expusieron los objetivos del estudio. Una vez otorgado el permiso se tuvo un primer contacto con los participantes a quienes se les explicó la intencionalidad del estudio y la importancia de su participación; de este modo, a las personas que voluntariamente desearon participar se les entregó el asentimiento y consentimiento informado para que sea firmado por sus padres y seguidamente por ellos. Posteriormente, se aplicaron los instrumentos en una sola jornada. La duración fue de 25 minutos. Con los resultados obtenidos se procedió a realizar los análisis estadísticos y se sintetizó toda la información en un informe final. 


\section{Análisis de datos}

El análisis de datos se llevó a cabo mediante el software IBM SPSS Stactistics Standard versión 25.0. Inicialmente se realizó un análisis descriptivo para cada variable en el que se evalúo la media, desviación estándar, puntaje mínimo y máximo. Posteriormente, se ejecutó la prueba de normalidad Kolmogorov-Smirnov para determinar la distribución de los datos. Teniendo en cuenta que los datos fueron no paramétricos se aplicó el coeficiente r de Spearman. Se estipuló que un valor $r$ cercano a 1 indica que la relación es positiva y fuerte; por el contrario, si el valor se aproxima a -1 representa una relación negativa y fuerte. Asimismo, si la relación esta próxima a 0.5 se considera que es una relación moderada y positiva, y si está cercano a -0.5 se trata de una relación moderada y negativa. Si el valor se aproxima a 0 refleja una correlación débil (Burbano y Valdivieso, 2016).

\section{Resultados}

La muestra quedó constituida por 350 estudiantes, de los cuales eran el 57,1\% (n=200) hombres y el 42,9 \% (n=150) mujeres. Sus edades oscilan entre los 13 y 18 años, con una media de 15,34. A nivel escolar el $50 \%(n=175)$ cursa educación básica secundaria, correspondiente a $6^{\circ}, 7^{\circ}, 8^{\circ}$ y $9^{\circ}$, y el otro $50 \%(n=175)$ cursa educación vocacional $10^{\circ}$ y $11^{\circ}$ de dos instituciones educativas públicas. En lo que confiere al sector de residencia el $82 \%(n=287)$ de los estudiantes viven en zonas urbana y el $18 \%(n=63)$ restante en zonas rurales. En la variable deprivación sociocultural se encontró una media de 18.74 y una desviación estándar de 6,60 en la escala total, siendo el puntaje mínimo de 2 puntos y el máximo de 40 puntos. A nivel general, se encontró que el 20,85 \% $(n=73)$ de participantes presenta un nivel alto de predisposición a la deprivación sociocultural, el 40,57 \% $(n=142)$ un nivel medio, y el 38,57 \% $(n=135)$ un nivel bajo.

Por otra parte, teniendo en cuenta que las dimensiones de la Escala de Deprivación Sociocultural no tienen la misma cantidad de ítems, para comparar las puntuaciones medias entre las mismas se dividió la puntuación media total de la dimensión por la cantidad de ítems que posee cada una de ellas. De esta forma, se evidenció que la dimensión apoyo social comunitario posee 
puntuaciones más elevadas (ver figura 1). Esto puede deberse a la deficiencia en las relaciones de los participantes con sus comunidades, al no percibirlas como una fuente de apoyo o ayuda real; lo anterior, puede derivarse de ineficientes procesos de integración, participación y apoyo en comunidades cada vez más individualizadas.

Por el contrario, se identificó que las variables del ámbito familia, tanto clima social familiar como dinámica familiar, presentan las puntuaciones más bajas respecto a los ámbitos escolar y comunitario. Esto implica que los participantes perciben una fuente de apoyo y bienestar más efectiva en la familia, pese a presentar dinámicas de conflictividad. Respecto a la variable de clima social escolar, la cual integra la calidad de los vínculos entre pares, las relaciones de respeto y colaboración entre docentes y estudiantes, la organización, claridad en las normas y apoyo en labores académicas, así como las dinámicas de competitividad, tiene una puntuación intermedia, lo que puede explicar la baja adherencia de los participantes con los procesos escolares.

A partir de lo anterior, puede identificarse la amplia incidencia del contexto comunitario en el desarrollo del individuo, entre tanto el barrio y la comunidad se constituyen en escenarios primordiales para la socialización secundaria, los cuales han venido siendo impactados por los procesos de individuación social que han afectado la percepción del sentido comunitario, marginando al individuo de la comunidad como fuente de apoyo social. En efecto, desde una perspectiva sistémica para minimizar el riesgo al aislamiento social y la falta de fuentes de estimulación adecuadas para el desarrollo, que terminen en la generación de sentimientos de inferioridad en los adolescentes, es necesario el fortalecimiento de las relaciones de reciprocidad entre los sistemas sociales nucleares (familia, escuela, comunidad), entre tanto, las deficiencias en el apoyo social comunitario disminuyen las fortalezas que podrían emerger de una positiva dinámica familiar.

\section{Figura 1}


Puntajes de las dimensiones de la Escala de Factores Predisponentes a la Deprivación Sociocultural

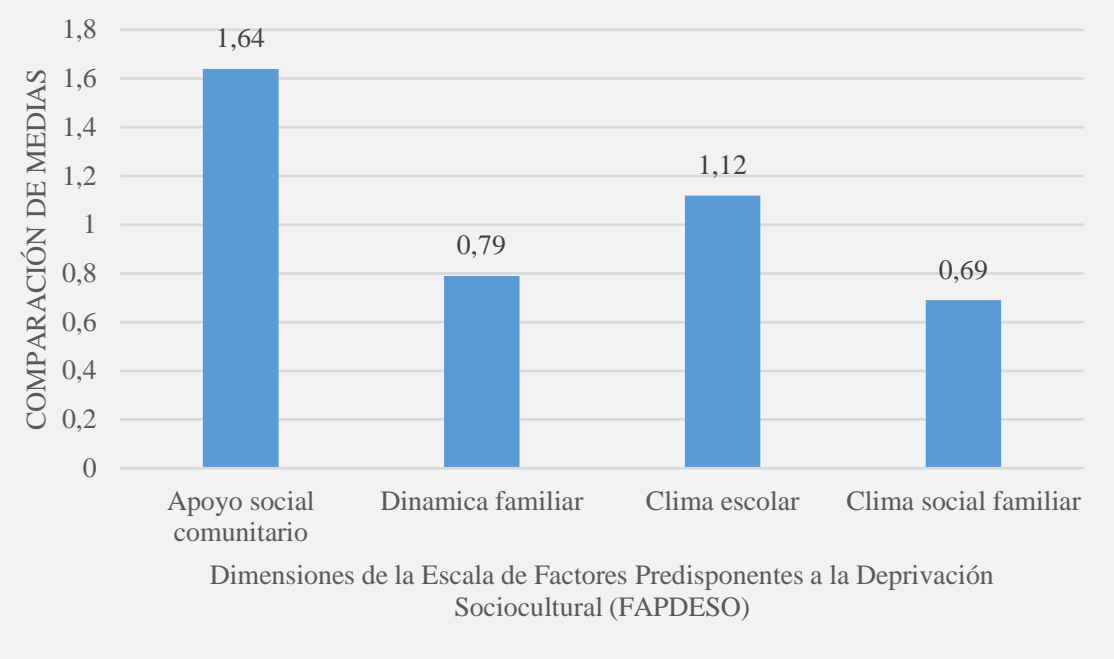

Nota: elaboración propia.

En lo que confiere a la variable de apoyo social, se encontró una media de 32.71 y una desviación estándar de 10,78 en la escala total de la escala, siendo el puntaje mínimo de 0 puntos y el máximo de 52 puntos. A nivel general, se encontró que el 7,71 \% (n=27) de participantes presenta un nivel alto de apoyo social, el 86,28 \% ( $\mathrm{n}=302)$ un nivel medio, y el $6 \%(\mathrm{n}=21)$ un nivel bajo. Desde otra perspectiva, para determinar la relación entre apoyo social y deprivación sociocultural en un grupo de adolescentes de la ciudad de Pasto, Colombia, se procedió a aplicar la prueba de normalidad Kolmogorov-Smirnov, encontrando un valor $p$ de ,005, lo que significa que los datos no se distribuyen de forma normal, por lo que se procedió a aplicar pruebas no paramétricas. De este modo, se aplicó la prueba Spearman mediante la cual se encontró una relación inversa estadísticamente significativa entre las variables (ver tabla 1).

\section{Tabla 1}

Correlación entre la deprivación sociocultural y el apoyo social

Rho de Spearman




\begin{tabular}{llr}
\cline { 2 - 3 } Total & Sig. (bilateral) &, 000 \\
FAPDESO & $\mathrm{N}$ & 350 \\
\hline
\end{tabular}

Nota: fuente elaboración propia.

**. La correlación es significativa en el nivel 0,01 (bilateral).

En relación con la tabla 1, se propone que a menor apoyo social mayor exposición a los factores predisponentes a la deprivación sociocultural; es decir, que cuando las relaciones comunitarias, familiares o escolares se perciben como deficientes por los adolescentes, estos son expuestos a factores predisponentes a la deprivación sociocultural, lo que podría acarrear un impacto negativo sobre el desarrollo social, emocional y cognitivo, como consecuencia de los deprivados entornos sociales, familiares o escolares que no coadyuvan en la facilitación de apoyo instrumental, informativo o afectivo, necesario para el desarrollo integral.

Posteriormente, se efectúo una correlación entre las dimensiones de la FAPDESO con el apoyo social (tabla 2), en la cual se encontró que todas las dimensiones tienen una relación negativa con el apoyo social. Se evidencia que el apoyo social comunitario es el que presenta una relación tendiente a moderada, en comparación con las demás dimensiones. Esto corrobora la importancia del estudio del apoyo social comunitario en el abordaje de la deprivación sociocultural.

\section{Tabla 2}

Asociación entre las dimensiones de la FAPDESO con el apoyo social

\begin{tabular}{lllllll}
\hline & & & \multicolumn{1}{c}{$\begin{array}{c}\text { Clima } \\
\text { social } \\
\text { familiar }\end{array}$} & $\begin{array}{c}\text { Apoyo social } \\
\text { comunitario }\end{array}$ & $\begin{array}{c}\text { Dinámicas } \\
\text { familiares }\end{array}$ & $\begin{array}{c}\text { Clima } \\
\text { escolar }\end{array}$ \\
\hline \multirow{2}{*}{$\begin{array}{l}\text { Rho de } \\
\text { Spearman }\end{array}$} & $\begin{array}{l}\text { Total } \\
\text { apoyo } \\
\text { social }\end{array}$ & $\begin{array}{l}\text { Coeficiente } \\
\text { de }\end{array}$ &,$- 364^{* *}$ &,$- 377^{* *}$ &,$- 225^{* *}$ &,$- 298^{* *}$ \\
\cline { 3 - 7 } & $\begin{array}{l}\text { Sig. } \\
\text { (bilateral })\end{array}$ &, 000 &, 000 &, 000 &, 000 \\
\cline { 2 - 7 } & $\mathrm{N}$ & 350 & 350 & 350 & 350 \\
\hline
\end{tabular}

Nota: Fuente: elaboración propia.

**. La correlación es significativa en el nivel 0,01 (bilateral).

Los anteriores resultados ponen de manifiesto que el apoyo social se constituye en una de las variables más relevantes al momento de predecir entornos sociales potencialmente deprivados, 
siendo, a su vez, el apoyo social uno de los ejes estructurales al momento de mitigar los efectos de la deprivación sociocultural.

\section{Discusión}

La deprivación sociocultural se ha constituido en un importante tema de interés al momento de comprender la influencia de los sistemas relacionales sobre la estructuración cognitiva. En el abordaje de los sistemas relacionales es indispensable, al momento de comprender cuáles son los factores que influyen o predisponen a los individuos a las manifestaciones de la deprivación sociocultural, analizar cómo están las fuentes de apoyo. Es así como diversos estudios han evaluado la influencia de variables como el clima escolar, el apoyo social comunitario, la dinámica y el clima familiar, en relación con manifestaciones de la deprivación sociocultural. Tal es el caso, en estudios de comportamientos impulsivos (Pérez et al., 2018), del procesamiento de la información (Rodríguez et al., 2018), de la calidad de vida (Molina et al., 2016) o de las pocas aspiraciones escolares (Fernández-González et al., 2015); sin embargo, lo han hecho de manera aislada, lo que reduce la percepción global de la incidencia de los sistemas nucleares sobre el desarrollo del individuo.

En este escenario, los resultados advierten una relación inversa estadísticamente significativa entre el apoyo social y los factores predisponentes a la DS, hallazgos similares a los encontrados por Orcasita y Uribe (2010), quienes analizan la influencia del apoyo social en determinados comportamientos de riesgo. Es así como tanto la calidad de las relaciones sociales como su funcionalidad, son indispensables al momento de comprender la influencia del medio social sobre el desarrollo del individuo, razón por la cual la dimensión de apoyo instrumental o emocional, como eje de facilitación de los entornos sociales y relaciones humanas, es fundamental al momento de estudiar los factores predisponentes a la deprivación sociocultural (Narváez, 2019), por cuanto, las deficientes fuentes de apoyo conllevan una serie de afectaciones sobre la socialización y desarrollo de los escolares.

Los resultados evidencian que la dimensión apoyo social comunitario posee puntuaciones más elevadas. Lo anterior, puede explicarse debido a los procesos de fragmentación comunitaria 
que minimizan la percepción de apoyo de los individuos (Narváez, 2016), por cuanto las condiciones comunitarias no proporcionan sentimientos de vinculación, situación que minimiza las provisiones expresivas o instrumentales proporcionadas por la comunidad o redes comunitarias (Gracia y Herrero, 2006). De este modo, el nivel de apoyo social comunitario influye en diversos factores de ajuste y bienestar psicológico, por cuanto la comunidad se constituye en un sistema potencial que, al contar con recursos de apoyo disponibles, podría compensar las carencias del individuo en la escuela o la familia. De esta forma, los individuos que se perciben más integrados a su comunidad perciben mayor apoyo social comunitario, lo que beneficia su salud mental y favorece su desarrollo (López y Sánchez, 2001).

Contrario al apoyo social comunitario, el factor de clima social familiar representa el puntaje más bajo, lo que se contrasta con que el $40.57 \%$ y el $38.57 \%$ de los participantes presenten niveles medio y bajo de predisposición a la deprivación sociocultural. El clima social familiar representa la dimensión más relevante al momento de visualizar el curso del desarrollo del adolescente, de allí que la conflictividad entre padres e hijos tendrá como consecuencia el aumento del malestar emocional y el estrés experimentado (Oliva, 2006); lo anterior, por cuanto los conflictos representan un aspecto duradero de la dinámica familiar que es menos sensible a los cambios en las relaciones familiares, lo cual lleva a los adolescentes a una disminución de la conectividad y, como consecuencia, de la percepción de apoyo (Stuart \& Jose, 2012); situaciones que exponen al individuo al riesgo de deprivación sociocultural.

En función a lo anterior, para el caso de la muestra en estudio, el que los participantes perciban un clima familiar positivo es consecuente con los bajos niveles presentados en la escala FAPDESO; situación contraria, a si las familias de los participantes se percibieran poco cohesionadas, con interacciones conflictivas y desorganización, dimensiones del clima social familiar que podrían determinar pautas desadaptativas y disminuidos recursos psicológicos (Rosales y Espinosa, 2009), escenario en el cual el clima social familiar, dentro de la escala, podría presentar niveles altos.

Frente al apoyo social, sólo el $7.71 \%$ de participantes presenta un nivel alto, y la mayor parte de ellos (86.28\%) se encuentra en nivel medio; tales puntuaciones explican las deficiencias encontradas en relación con el apoyo social comunitario, factor predisponente a la deprivación sociocultural que puntúa más alto en la escala FAPDESO. Teóricamente el apoyo social se 
constituye en uno de los componentes psicológicos más influyentes en el individuo (Bravo y Fernández, 2003; Jiménez, 2006); de allí que la baja percepción de apoyo social, en sus dimensiones informacional, instrumental o emocional, en los ámbitos familiar, comunitario y escolar, predispongan al individuo a sufrir una serie de carencias que podrían detonar en manifestaciones de deprivación sociocultural (Narváez, 2019). Estudios del apoyo social y las conductas delictivas (Bravo y Fernández, 2003), del apoyo social y satisfacción con la vida (Novoa y Barra, 2015) o del apoyo social en adolescentes con ansiedad y depresión (Dávila et al., 2011) dan cuenta de la relevancia del apoyo social como factor protector frente a eventos y estresores psicosociales que pueden influir, de manera negativa, en la vida de las personas, desencadenando manifestaciones como la deprivación sociocultural. Situación que da cuenta de la relación estadísticamente significativa entre las variables en estudio, lo que implica que a menor apoyo social mayores factores predisponentes a la deprivación sociocultural.

Por otra parte, se encontró que el 86,28 \% $(\mathrm{n}=302)$ de los sujetos presentan un nivel medio en apoyo social, lo que explica las puntuaciones en los factores predisponentes a la deprivación sociocultural, donde el 40,57\% $(\mathrm{n}=142)$ presenta un nivel medio y el 38,57 \% $(\mathrm{n}=135)$ un nivel bajo. El nivel medio en apoyo social podría indicar que alguna de las fuentes de apoyo social, en los ámbitos escolar, familiar o comunitario, no está aportando ayuda instrumental, emocional o informacional a los adolescentes, información que se corrobora cuando los factores de apoyo social comunitario y clima social escolar puntúan más bajos en la escala FAPDESO. Lo que podría explicar los hallazgos de Cheng et al. (2014), para quienes los adolescentes que viven en zonas económicamente desfavorables registran altos niveles de depresión, como consecuencia, de bajo nivel de apoyo social en las familias y barrios, lo que aumenta los niveles de estrés y angustia.

Al respecto, estudios como los de Cifuentes (2015) dan cuenta de la percepción de un grupo de estudiantes frente a la escuela, y la incidencia de la percepción de las carencias escolares sobre los niveles de bienestar reportados; entre los factores influyentes en dicha percepción están la calidad de las relaciones y los estados anímicos de los docentes, el nivel de convivencia entre pares, así como las normas de higiene en el cuidado del entorno. Hallazgos que podrían contrastarse con los de Londoño et al. (2018), donde los participantes en un estudio de convivencia, en la variable interpersonal, señalan altos niveles de desconfianza con los maestros, baja disponibilidad de ayuda instrumental o emocional, así como manifestaciones de violencia y baja 
participación del alumnado en la construcción de normas de convivencia, o con el aporte de Daily et al. (2019), quienes identifican la relevancia del clima escolar en la promoción de la salud mental positiva y el logro académico en las escuelas. Las anteriores, dimensiones vinculadas al factor de apoyo social escolar de la escala FAPDESO.

Respecto al apoyo social comunitario, diversos estudios dan cuenta de la influencia de la comunidad y el sentido de comunidad sobre el bienestar de los individuos; investigadores como Cueto et al. (2016) señalan que la comunidad, como escenario de la construcción de relaciones humanas, favorece tanto la cohesión y la percepción de interdependencia, así como la satisfacción de necesidades grupales, psicológicas y colectivas; siendo el apoyo social uno de los factores más importantes al momento de evaluar la capacidad de las redes comunitarias en el suministro de ayuda a sus miembros. De igual manera, Espinosa et al. (2016) sustentan que uno de los efectos de las redes sociales y la comunidad sobre el bienestar subjetivo del individuo está asociada con la disminución de las consecuencias negativas o de eventos potencialmente estresantes. Lo anterior, por cuanto el grupo comunitario favorece el sentido de aceptación, autovaloración, afiliación y contacto, lo que facilita la capacidad de apoyo y el soporte del individuo con su sistema comunitario. Situación contraria, a lo encontrado en la muestra del estudio, donde un alto porcentaje de los participantes señalan no contar con fuentes de ayuda comunitarias.

En el ámbito familiar, en correspondencia con el puntaje en la escala de factores predisponentes de deprivación sociocultural, donde sus dos factores puntúan más bajo, podría inferirse que a ello responde el nivel medio en apoyo social; es decir, que la mayor parte de los participantes que percibe un clima familiar positivo y una dinámica familiar cohesionada, perciben a su vez que la familia es una importante fuente de apoyo social, escenario que genera contención hacia las puntuaciones de factores predisponentes de deprivación sociocultural, donde el 20,85\% $(n=73)$ puntúan alto; caso contrario sería si las puntuaciones de apoyo social en la familia fueran negativas. En ese escenario, se destaca que el clima social familiar, afecto y comunicación son tres de los principales factores para el bienestar psicológico del adolescente. Por tanto, las niñas y niños que perciben más afecto de sus padres y disfrutan de una buena relación con ellos muestran mejor desarrollo psicosocial, mayor bienestar emocional y mejor ajuste del comportamiento (Cerezo et al., 2018). 
En relación con la incidencia de la familia en las variables en estudio, Jiménez et al. (2005) plantean a la dinámica y clima familiar como dos importantes variables al momento de comprender la conducta delictiva en los adolescentes; esta una de las manifestaciones de la deprivación sociocultural. Entre los factores de riesgo identificados está la baja cohesión, el conflicto, las pobres interacciones entre padres e hijos, el estilo de socialización negligente y la disciplina coercitiva. Paralelamente, autores como Parke (2004) señalan a la familia como una fuente potente de recursos, que permite a los adolescentes obtener apoyo social al momento de afrontar las tensiones y transiciones evolutivas. Así las cosas, la disponibilidad de apoyo social actúa como factor protector del individuo, minimizando el riesgo a los factores predisponentes a la deprivación sociocultural, mientras su ausencia es un factor de riesgo que podría detonar el desajuste psicosocial, y explicar la situación de desventaja social de individuos que no han contado con fuentes de apoyo en los ámbitos familiar, comunitario o escolar.

\section{Conclusiones}

La deprivación sociocultural se ha relacionado con aquellos contextos en los que ha existido poca o escasa estimulación o fuentes de apoyo instrumental, emocional e informacional, lo cual influye en el normal desarrollo cognitivo, físico o emocional de los individuos, cuya consecuencia ha sido un estado de desventaja observable en relación con los pares de su mismo contexto. Entre los factores predisponentes a dicho fenómeno se encuentran el clima familiar, el clima social escolar, el apoyo social comunitario y la dinámica familiar, los cuáles, de acuerdo con los resultados de este artículo, muestran una relación con el apoyo social. A mayor percepción de apoyo social menores factores predisponentes a la deprivación sociocultural.

Se propone que, dentro de los factores predisponentes a la deprivación sociocultural, el clima social familiar presentó el puntaje más bajo; esto significa que aquellos núcleos familiares en los que existen relaciones positivas de ayuda y apoyo mutuo dinamizan factores protectores para contrarrestar los factores predisponentes a la deprivación sociocultural. Por el contrario, se identificó que el apoyo social comunitario es la dimensión que puntúo más alto en la escala FAPDESO, lo que pone en evidencia la necesidad de mejorar los niveles de integración y participación del individuo en la comunidad, con el fin de que esta se constituya en una fuente 
importante de recursos, lo que llevaría al incremento de percepción de apoyo comunitario y minimizaría el riesgo de deprivación sociocultural.

Se resalta el papel del apoyo social, el cual, al tener una relación inversa estadísticamente significativa con los factores predisponentes de la deprivación sociocultural, pasa a ser un factor protector que permite el desarrollo de las condiciones relacionales afectivas o instrumentales para que el sujeto pueda desenvolverse en los diferentes sistemas en los que está inmerso, de acuerdo con los ámbitos familiar, educativo y escolar. En este contexto, como estudios futuros, es importante el desarrollo de propuestas investigativas que logren medir el efecto del fortalecimiento del apoyo social sobre los factores socioambientales asociados a los contextos sociales deprivados, analizando tanto la incidencia sobre el desarrollo integral de los individuos como el impacto en las capacidades familiares y comunitarias; respecto al entorno educativo, debe promoverse el diseño de propuestas de intervención desde programas o estrategias orientadas al desarrollo de procesos de mediación del aprendizaje, donde se permita una estimulación adecuada, haciendo de la escuela una real fuente de apoyo social que contribuya a la superación de los efectos de la DS en el desarrollo del individuo.

\section{Referencias}

Aranda, C., y Pando, M. (2013). Conceptualización del apoyo social y las redes de apoyo social. Revista de Investigación en Psicología, 16(1), 233-245. https://revistasinvestigacion.unmsm.edu.pe/index.php/psico/article/view/3929/3153

Barcelata, B., Granados, A., y Ramírez, A. (2013). Correlatos entre funcionamiento familiar y apoyo social percibido en escolares en riesgo psicosocial. Revista Mexicana de Orientación Educativa, $10(24)$ $65-70$. http://pepsic.bvsalud.org/scielo.php?script=sci_arttext\&pid=S1665$\underline{75272013000100008 \& \operatorname{lng}=\mathrm{pt} \& \mathrm{tlng}=\mathrm{es}}$

Barello, A., Palmucci, V., y Schuager, N. (2007). Reflexión Teórica: De novatos a expertos del pensar: La teoría de la modificabilidad estructural cognitiva. Revista Tempus, 4(3). https://www.xpsicopedagogia.com.ar/de-novatos-a-expertos-del-pensar-la-teoria-de-la$\underline{\text { modificabilidad-estructural-cognitiva.html }}$ 
Bravo, A., y Fernández, J. (2003). Las redes de apoyo social de los adolescentes acogidos en residencias de protección. un análisis comparativo con población normativa. Psicothema, 15(1), 136-142. http://www.psicothema.com/psicothema.asp?id=1035

Burbano, V., y Valdivieso, M. (2016). Inferencia Estadística Básica, apoyo al estudio independiente. UPTC.

Cabezas, A. (2017). Aspectos a considerar frente al concepto de inteligencia en niños privados socioculturalmente: la importancia de las evaluaciones dinámicas. Tabanque, (30), 239248. https://doi.org/10.24197/trp.30.2017.239-248

Cedillo, I. (2010). El Aprendizaje Mediado y las Operaciones Mentales de Comparación y Clasificación (Trabajo de grado de maestría). Universidad de Cuenca.

Cerezo, F., Ruiz, C., Sánchez, C., \& Arense, J. (2018). Dimensions of parenting styles, social climate, and bullying victims in primary and secondary education. Psicothema, 30 (1), 5965. https://doi.org/10.7334/psicothema2016.360

Cheng, Y., Li, X., Lou, C., Sonenstein, F. L., Kalamar, A., Jejeebhoy, S., Delany-Moretlwe, S., Brahmbhatt, H., Oluwafunmilola Olumide, A., \& Ojengbede, O. (2014)._The Association Between Social Support and Mental Health Among Vulnerable Adolescents in Five Cities: Findings from the Study of the Well-Being of Adolescents in Vulnerable Environments. Journal of Adolescent Health, 55(6), S31-S38. https://doi.org/10.1016/j.jadohealth.2014.08.020

Cifuentes, J. E. (2015). Una mirada a la escuela desde las voces de unos estudiantes del sector rural. Revista Virtual Universidad Católica del Norte, (45), 159-172. http://revistavirtual.ucn.edu.co/index.php/RevistaUCN/article/view/664/1195

Cueto, R., Espinosa, A., Guillén, H., y Seminario, M. (2016). Sentido de Comunidad Como Fuente de Bienestar en Poblaciones Socialmente Vulnerables de Lima, Perú. Psykhe, 25(1), 1-18. https://doi.org/10.7764/psykhe.25.1.814

Daily, S. M., Mann, M. J., Kristjansson, A. L., Smith, M. L., \& Zullig, K. J. (2019). School Climate and Academic Achievement in Middle and High School Students. Journal of School Health, 89(3), 173-180. https://doi.org/10.1111/josh.12726 
Dávila, A., Ruiz, R., Moncada, L., y Gallardo, I. (2011). Niveles de ansiedad, depresión y percepción de apoyo social en estudiantes de Odontología de la Universidad de Chile. Revista de Psicología, 20(2), 147-172. https://doi.org/10.5354/0719-0581.2012.17937

Espinosa, A., Freire, S., y Ferrándiz, J. (2016). Identificación colectiva y bienestar en una comunidad rural de la costa norte del Perú. Revista de Psicología, 34(1), 201-219. http://revistas.pucp.edu.pe/index.php/psicologia/article/view/14563

Estévez, E., Murgui, S., Musitu, G., y Moreno, D. (2008). Clima familiar, clima escolar y satisfacción con la vida en adolescentes. Revista Mexicana de Psicología, 25(1), 119-128. https://www.uv.es/ lisis/david/mexicana.pdf

Falla, S., Hermelín, D., y Aguirre, C. (2016). Conectar comunidades para construir sentidos sociales en torno al conocimiento. Trilogía Ciencia Tecnología Sociedad, 8(15), 57-68. https://doi.org/10.22430/21457778.400

Fernández-González, L., González-Hernández, A., y Trianes-Torres, MV (2015). Relaciones entre estrés académico, apoyo social, optimismo-pesimismo y autoestima en estudiantes universitarios. Revista Electrónica de Investigación en Psicología Educativa, 13(1), 112130. https://www.redalyc.org/articulo.oa?id=2931/293139632005

Fuica, P., Lira, J., Alvarado, K., Araneda, C., Lillo, G., Miranda, R., Tenorio, M., y Pérez-Salas, C. (2014). Habilidades Cognitivas, Contexto Rural y Urbano: Comparación de Perfiles WAIS-IV en Jóvenes. Terapia Psicológica, 32(2), 143-152. https://dx.doi.org/10.4067/S0718-48082014000200007

Gallego, A., Pino, J., Álvarez, M., Vargas, E., y Correa, L. (2019). La dinámica familiar y estilos de crianza: pilares fundamentales en la dimensión socioafectiva. Hallazgos, 16(32), 131150. https://doi.org/10.15332/

Gálvez, M., Álvarez, R., y Bernabé, R. (2018). Relación entre el clima social, familiar y escolar y el rendimiento académico de los estudiantes del $5^{\circ}$ de secundaria del colegio Luis Alberto Sánchez del distrito Gregorio Albarracín de Tacna, año 2016. Ciencia y Desarrollo, 21(2), 77-87. http://revistas.uap.edu.pe/ojs/index.php/CYD/article/view/1711/1649

García, A. (2003). Procesos cognitivos en la composición escrita de niños con deprivación sociocultural. Revista Interuniversitaria de Formación del Profesorado, (46), 179-191. http://e-spacio.uned.es/fez/eserv/bibliuned:20338/procesos_cognitivos.pdf 
Gómez González, S., Riverón Hernández, K., Jay Griñán, M., y Madrigal León, D. Y. (2017). Prácticas culturales en jóvenes universitarios cubanos. Trilogía Ciencia Tecnología Sociedad, 9(16), 101-111. https://doi.org/10.22430/21457778.177

Gracia, E., y Herrero, J. (2006). La comunidad como fuente de apoyo social: evaluación e implicaciones en los ámbitos individual y comunitario. Revista Latinoamericana de Psicología, $38(2)$, 327-342. http://pepsic.bvsalud.org/scielo.php?script=sci_arttext\&pid=S0120$\underline{05342006000200007 \& \operatorname{lng}=\mathrm{pt} \& \operatorname{tlng}=\mathrm{es}}$

Hernández, R., Fernández, C., y Baptista, P. (2010). Metodología de la investigación. McGrawHill.

Hombrados-Mendieta, I., \& Castro-Travé, M. (2013). Social support, social climate, and the perception of conflict in an intercultural educational context. Anales de Psicología, 29(1), 108-122. https://doi.org/10.6018/analesps.29.1.123311

Jiménez, D. (2009). Deprivación Sociocultural. Revista Temas para la Educación, (5), 1-10. https://www.feandalucia.ccoo.es/docuipdf.aspx?d=6391\&s=

Jiménez, T. I. (2006). Familia y problemas de desajuste en la adolescencia: el papel mediador de los recursos psicosociales (Tesis de doctorado). Universidad de Valencia.

Jiménez, T., Musito, G., y Murgui, S. (2005). Familia, apoyo social y conducta delictiva en la adolescencia: efectos directos y mediadores. Anuario de Psicología, 36(2), 181-195. https://www.uv.es/ lisis/gonzalo/7_musitu.pdf

Londoño, L. F., Estrada, E. P., y Alvarado, J. A. (2018). Caracterización de las problemáticas de convivencia de los estudiantes de secundaria de los colegios lasallistas, del distrito área metropolitana Medellín, en los componentes: socio demográfico y de convivencia. Revista Virtual Universidad Católica del Norte, (54), 5-17. https://revistavirtual.ucn.edu.co/index.php/RevistaUCN/article/view/977/1428

López, A., y Sánchez, E. (2001). Estructura social, apoyo social y salud mental. Psicothema, 13(1), 17-23. http://www.psicothema.com/psicothema.asp?id=408

López, R., y Herrera, L. (2019). Apoyo comunitario percibido en jóvenes y voluntariado. Revista INFAD de Psicología. International Journal of Developmental and Educational Psychology, 4(1), 117-126. https://doi.org/10.17060/ijodaep.2019.n1.v4.1524 
Lundber, I. (1998). Why is learning to read a hard task for some children? Scandinavian Journal of Psychology, 39(3), 155-157. https://doi.org/10.1111 / 1467-9450.393071

Matud, M. P. (1998): Investigación del estrés y su impacto en la salud de las mujeres en Canarias. Instituto Canario de la Mujer.

Mena, E., Kroll, L. E., Maier, W., \& Bolte, G. (2018). Gender inequalities in the association between area deprivation and perceived social support: a cross-sectional multilevel analysis at the municipality level in Germany. BMJ Open, 8(4), 1-9. https://doi.org/10.1136/bmjopen-2017-019973

Molina, R., Sepúlveda, R., Carmona, R., Molina, T., y Mac-Ginty, S. (2016). Calidad de vida relacionada con la salud en estudiantes universitarios de primer año. Revista Chilena de Salud Pública, 20(2), 101-112. https://revistasaludpublica.uchile.cl/index.php/RCSP/article/view/44928

Narváez, J. (2016). Escenarios móviles y centros de contacto, una experiencia significativa en la dinamización del apoyo social. Rev Univ. Salud, 18(2), 312-324. http://www.scielo.org.co/pdf/reus/v18n2/v18n2a12.pdf

Narváez, J. (2019). Manifestaciones de la deprivación sociocultural y la violencia urbana. Editorial Universidad de Nariño.

Novoa, C., y Barra, E. (2015). Influencia del apoyo social percibido y los factores de personalidad en la satisfacción vital de estudiantes universitarios. Terapia Psicológica, 33(3), 239-245. https://dx.doi.org/10.4067/S0718-48082015000300007

Oliva, A. (2006). Relaciones familiares y desarrollo adolescente. Anuario de Psicología, 37(3), 209-223. https://www.redalyc.org/articulo.oa?id=970/97012834001

Orcasita, L., y Uribe, A. (2010). La importancia del apoyo social en el bienestar de los adolescentes. Psychologia: Avances de la Disciplina, 4(2), 69-82. https://revistas.usb.edu.co/index.php/Psychologia/article/view/1151/943

Parke, R. D. (2004). Development in family. Annual Review of Psychology, (55), 365-399. https://www.annualreviews.org/doi/abs/10.1146/annurev.psych.55.090902.141528

Pérez, G., Rubio, L., y Medina, B. (2018). Papel moderador de los estilos parentales en la relación entre la impulsividad y el consumo de alcohol en una muestra de adolescentes españoles. Revista de Psicopatología y Psicología Clínica, 23(1), 47-57. 
http://www.aepcp.net/arc/Revista\%20de\%20Psicopatologia\%20y\%20Psicologia\%20Clin ica_Vol.23(1).2018_Parte6.pdf

Reyes, V., Merino, T., Villavicencio, L., y Mendoza, C. (2019). El Clima Social Familiar y la Agresividad en Educación Primaria: un estudio de caso en Guayaquil - Ecuador. Research, Society and Development, $\quad 8(11), \quad 1-20$. https://dialnet.unirioja.es/servlet/articulo?codigo=7164822

Rodríguez, C., Ramírez, R., Fernández, M., y Bustos, C. (2018). Capital Psicológico, Desempeño Contextual y Apoyo Social del Trabajo en Salud Primaria. Journal of Work and $\begin{array}{lll}\text { Organizational } & \text { Psychology, } & \text { 34(3), }\end{array}$ https://journals.copmadrid.org/jwop/art/jwop2018a15

Rosales, C., y Espinosa, M. (2009). La percepción del clima familiar en adolescentes miembros de diferentes tipos de familias. Facultad de Estudios Superiores Iztacala, 10(1), 64-71. https://www.researchgate.net/publication/237032729_La_Percepcion_del_Clima_Familia $\underline{\text { r_en_Adolescentes_Miembros_de_Diferentes_Tipos_de_Familias }}$

Ruiz, C., y Merino, D. (2009). La experiencia escolar de los hijos de inmigrantes marroquíes en los Centros de Educación Secundaria Obligatoria. Revista Electrónica Interuniversitaria de Formación del Profesorado, 12(3), 87-97. https://dialnet.unirioja.es/servlet/articulo?codigo=3086539

Sánchez, M. (2010). Alumnos con deprivación sociocultural ¿Qué hacemos? Revista de Innovación $\quad y \quad$ Experiencias $\quad$ Significativas, $\quad$ (16), 1-10. https://pdfs.semanticscholar.org/b5f2/05a1896a0fcec1f3ef618eca56ac6aee734b.pdf

Stuart, J., \& Jose, P. E. (2012). The influence of discrepancies between adolescent and parent ratings of family dynamics on the well-being of adolescents. Journal of Family Psychology, 26(6), 858-868. https://dx.doi.org/10.1037/a0030056

Suarez, P., y Vélez, M. (2018). El papel de la familia en el desarrollo social del niño: una mirada desde la afectividad, la comunicación familiar y estilos de educación parental. Psicoespacios, $12(20)$, 173-197. http://revistas.iue.edu.co/revistasiue/index.php/Psicoespacios/article/view/1046 


\section{Relación entre}

factores

Thomas, S. A., \& Shihadeh, E. S. (2013). Institutional isolation and crime: The mediating effect of disengaged youth on levels of crime. Social Science Research, 42(5), 1167-1179. https://dx.doi.org/10.1016/j.ssresearch.2013.03.009

Uribe, A. F. (2016). Salud sexual, apoyo social y funcionamiento familiar en universitarios. Revista Virtual Universidad Católica del Norte, (49), 206-229. http://revistavirtual.ucn.edu.co/index.php/RevistaUCN/article/view/805/1325

Villalobos, F. (2009). Ideación suicida en jóvenes: formulación y validación del modelo integrador explicativo en estudiantes de educación secundaria y superior (Tesis de doctorado). Universidad de Granada.

Zambrano, C., y Almeida, E. (2017). Clima social familiar y su influencia en la conducta violenta en los escolares. Revista Ciencia UNEMI, 10(25), 97-102. https://pdfs.semanticscholar.org/b5f2/05a1896a0fcec1f3ef618eca56ac6aee734b.pdf 